\title{
Dietary habits and cardiovascular disease risk in middle-aged and elderly populations: a review of evidence
}

This article was published in the following Dove Press journal:

Clinical Interventions in Aging

13 July 2009

Number of times this article has been viewed

\author{
Eleni Tourlouki \\ Antonia-Leda Matalas \\ Demosthenes B Panagiotakos \\ Department of Nutrition \\ Science - Dietetics, Harokopio \\ University, Athens, Greece
}

\begin{abstract}
Background: The proportion of elderly is increasing worldwide. This trend is paralleled by an increase in chronic diseases, such as cardiovascular disease. A limited number of studies have investigated the effect of diet on cardiometabolic risk factors (such as hypertension, hypercholesterolemia, diabetes, obesity) in older populations, despite the fact that diet plays a significant role in elderly health. In this review, a summary of studies that have evaluated the effect of dietary habits on cardiovascular disease risk in elderly populations is presented.
\end{abstract}

Methods: A search was made in available databases (PubMed and Scopus) looking for results from observational studies and clinical trials that assessed dietary habits on cardiovascular disease risk in elderly populations ( $>65$ years old). Studies during the last decade were retrieved and summarized.

Results: All eight of the reviewed observational studies and all three reviewed clinical trials performed in elderly populations reported an inverse association between healthy dietary patterns with cardiovascular disease risk and its predisposing markers.

Conclusion: Dietary intervention strategies should be implemented in older adults, in order to prevent cardiovascular disease morbidity and mortality, and improve quality of life.

Keywords: diet, cardiovascular disease, risk factors, elders

Globally, cardiovascular disease (CVD) is estimated to be the leading cause of mortality and loss of disability-adjusted life years. ${ }^{1-3}$ The World Health Organization (WHO) estimates that roughly $30 \%$ of global deaths result from CVD. ${ }^{4}$ In the European Union, CVD caused nearly half of all deaths (42\%) and cost the EU economy $€ 169$ billion a year. ${ }^{5}$ Similarly, CVD has increasingly affected low-income and middle-income countries. For example, in India and China, CVD is more abundant than in all the economically developed countries in the world put together. ${ }^{1}$ The global rise in CVD is congruent with a similar rise in the elderly population. According to a policy framework prepared by WHO, the proportion of people over the age of 60 is growing faster than any other age group. ${ }^{6}$ They go on to state that from 1970 to 2025 a growth of $223 \%$ is expected in older persons. Additionally, WHO estimates forecast that by 2025 , 120 countries will reach total fertility rates below the replacement level compared to a mere 22 countries in 1975 . The percentage of elderly people, in Japan and the United States, is expected to double in just 30 years; similarly, a threefold increase is likely to occur during the same period in the proportion of older people in China. ${ }^{7}$ This change in the age distribution of the world's population can be mainly attributed to the medical advancements of the twenty-first century leading to prolonged life
Correspondence: Eleni Tourlouki

El. Venizelou 70, Kalithea, Attica,

17671 , Greece

Tel +302108040119

Fax +302109600719

Email htourloukis@hotmail.com 
expectancy. Consequently, in the 1900 s, most countries in the world experienced a decline in infectious and parasitic diseases, a reduction in maternal and infant mortality, and improved nutrition. ${ }^{8}$ The United Nations depicts the growing numbers of elders worldwide in a global population pyramid. ${ }^{6}$ It illustrates the population in 2002 and how it is expected to evolve by 2025; the proportion of elderly is on the rise while the proportion of youth (0-20 years) is decreasing. This increase in life expectancy, and consequently, the aging population, is staggering posing tremendous challenges in disease burden, particularly, chronic diseases such as obesity, diabetes, cancer and CVD. Nearly $60 \%$ of the 56.5 million total reported global deaths were contributed to chronic diseases; approximately half are attributed to CVD (calculated in 2001). ${ }^{1}$ Accordingly, research must account for this epidemiological and demographic transition. Studies need to determine cost-effective interventions for managing these diseases in older populations.

\section{Diet and the elderly}

The role of diet in the prevention and control of morbidity and premature mortality due to non-communicable diseases has been well established by the vast population-based epidemiological studies carried out during the last decade. ${ }^{1,2}$ This growing awareness of preventive nutrition should underpin most interventions in order to decrease diet-related diseases such as CVD, and the substantial burden placed on health care, social and family structures. As mentioned above, elders, and particularly the very old ( $>85$ years), are most likely to suffer from chronic diseases. Not only does biological capacity decline with normal aging, but a range of experiences and behaviors throughout life dictate CVD risk. $^{2}$ For example, lack of physical activity in earlier years may negatively affect health status, while isolation and depression in later life may reduce appetite and increase the risk of nutrient deficiency. Furthermore, existing health conditions and malnutrition may exacerbate these illnesses. An increased risk of morbidity and mortality is associated with malnutrition in the elderly. The important role of diet in the prevention of disability and premature death in the aged is a well-recognized and accepted means of geriatric care. The American Heart Association has focused on preventative nutrition in elders. They recognize that a Mediterraneantype diet has notable effects on the CVD prognosis and have recommended that populations follow a similar diet (Step 1 Diet). ${ }^{9}$ Researchers at Tufts University have designed a Modified MyPyramid for Older Adults that corresponds to the new United States Department of Agriculture (USDA) food pyramid. ${ }^{10}$ This modified version includes icons representing six food categories and emphasizes the need to consume fluids and engage in adequate exercise. ${ }^{11}$ Therefore, adopting a healthy lifestyle, including a diet that adheres to healthy eating guidelines, can postpone morbidity and mortality from CVD, encourage healthy aging and regulate the disproportionate amount of health care resources this sector of the population requires.

In addition, research has predominantly focused on assessing single nutrients instead of overall dietary patterns like the Mediterranean, Western, or prudent diet. The difficulty with this type of analysis is that individuals consume a variety of foods and the effects of a whole diet may be greater than the sum of its parts. ${ }^{12}$ For example, the Mediterranean diet is recognized for its cardioprotective properties. However, the effect of the diet on mortality risk cannot be solely contributed to one component. ${ }^{12}$ Likewise, the unknown or unmeasured synergistic effects of specific foods on the investigated outcome may produce further obscurities when taking a single-food approach.

The current knowledge of preventive nutrition on CVD risk was mainly derived from various observational and clinical trials differing in study design, sample size and ethnic groups. This review focuses on studies that investigated food habits on CVD risk in various middle aged and elderly populations (Table 1).

\section{A summary of observational/ epidemiologic studies The Seven Countries Study}

Low rates of CVD, observed around late 1960s and 1970s, in the Mediterranean basin was one of the main findings of the historical Seven Countries Study. ${ }^{13}$ Particularly, this was the first study that investigated, among other factors, the relationship between eating habits and long-term incidence and mortality (through a 25-year follow-up) from coronary heart disease (CHD) and stroke, in differing middle-aged populations and cultures. ${ }^{14}$ Sixteen cohorts from seven countries (USA, Finland, The Netherlands, Italy, former Yugoslavia, Greece, and Japan) examined dietary information and vital status of 12,763 men (aged 40-59). Large variations, about a 10 -fold difference, in age-adjusted 25 -year death rates were reported between cohorts. ${ }^{13}$ The latter raised many questions; death rates varied greatly from East Finland exhibiting 268 per 1,000 (26.8\%), in comparison to 25 per 1,000 (2.5\%) observed in Crete. Analysis of the dietary information strongly correlated saturated fat with incidence and morality rates of CHD. This was one of the 


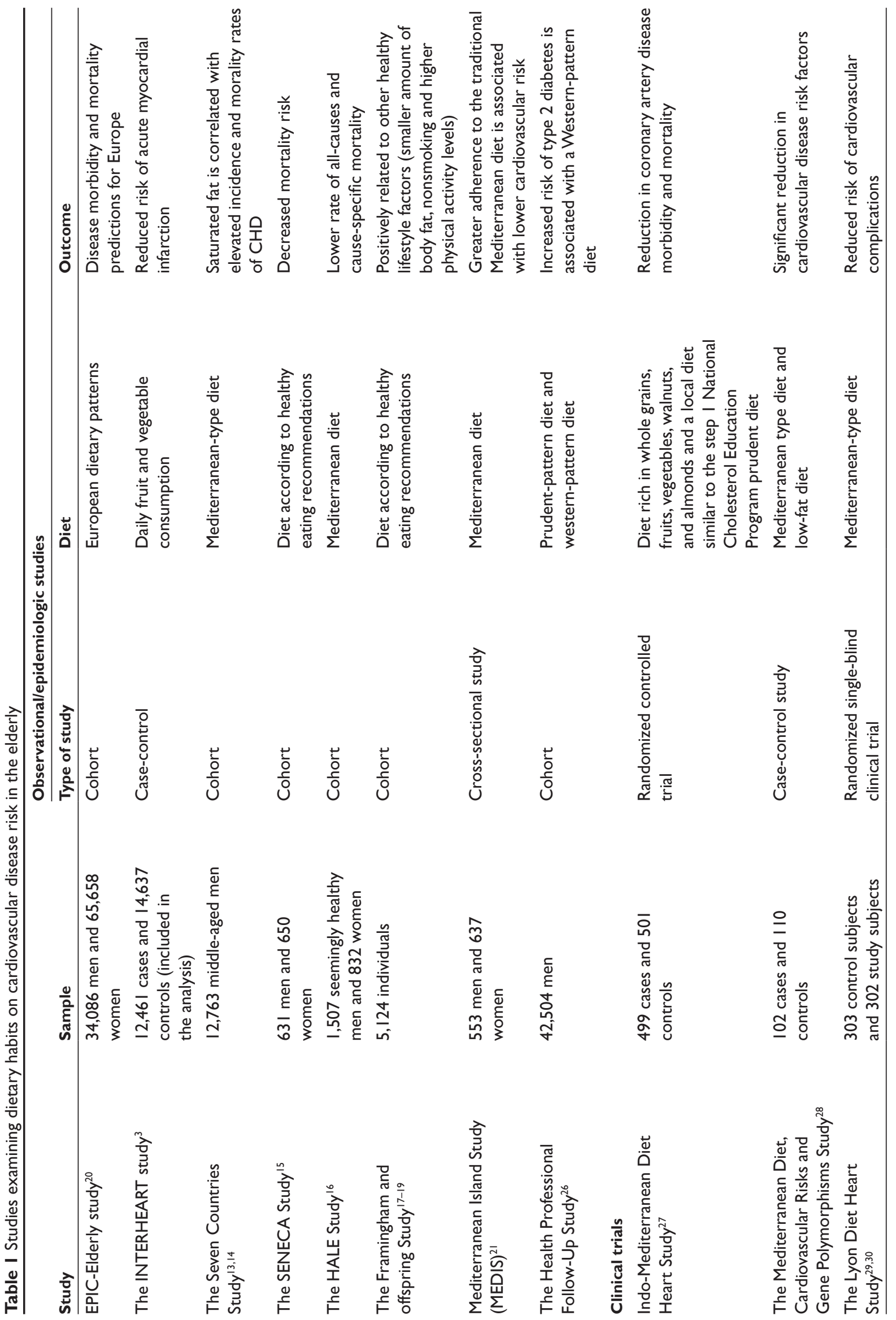


major findings of the Study, as differences in types of fat emerged. Disparities in rates of CHD existed; however, intakes of total fat as a percentage of total energy intakes did not vary greatly amongst countries. The effects of saturated and unsaturated fats on CHD mortality surfaced subsequent to this study. Dairy fat and lard (saturated fat) were consumed in northern Europe, the highest risk area; whereas, in southern Europe (the lowest risk area), fat was predominately from olive oil (unsaturated fat). Furthermore, Menotti and colleagues ${ }^{14}$ examined the association of simple food-groups and their combinations with 25-year mortality from CHD. Similar findings were discovered. Diets rich in butter, dairy products and other animal products (usually rich in saturated fatty acids and cholesterol) were associated high rates of $\mathrm{CHD}$. In contrast, food consumption patterns high in cereals, legumes, vegetable products, fish, oils and wine were associated with low or relatively low mortality rates from CHD. Overall, vegetable foods combined (excluding alcohol) were inversely correlated with CHD death rates; whereas, animal foods combined were directly correlated. Greece and Japan experienced the fewest death rates caused by CHD; USA, Finland, and The Netherlands, experienced the most. These findings suggested that dietary patterns are an important determinant of CHD risk, and reveal the Mediterranean diets' cardioprotective effect.

\section{The SENECA study}

The Survey in Europe on Nutrition and the Elderly: a Concerted Action (SENECA) began with baseline measurements in 1988 and 1989, and consisted of a 10-year survival follow-up. Study participants were 70-75 years old and born between 1913 and $1918 .{ }^{15}$ The study population included 631 men and 650 women from Belgium, Denmark, Italy, The Netherlands, Portugal, Spain and Switzerland. Dietary intake, health status, and lifestyle habits were obtained. The study investigated the effects of nonsmoking, being physically active, and having a high-quality diet (a diet that adheres to healthy eating guidelines) on survival. Throughout the study period, roughly half of the male population and three quarters of the female population survived. Data analysis revealed that for all study participants an increasing number of unhealthy lifestyle behaviors were related to a higher mortality rate $(\mathrm{p}<0.001)$. The relative risk of mortality for men with a low-quality diet was 1.25 (95\% confidence interval [CI]: $0.93,1.68)$, the relative risk associated with physical inactivity was 1.36 (95\% CI: 1.08, 1.73), and the relative risk for smoking was 2.06 (95\% CI: 1.63, 2.60). Similarly, the relative risk of mortality for women with a low-quality diet was 1.26 (95\% CI: $0.88,1.81)$, the risk associated with physical inactivity was 1.75 (95\% CI: 1.29, 2.39), and the risk for smoking was 1.75 (95\% CI: 1.29, 2.39). For all subjects with three unhealthy lifestyle behaviors (assuming no interaction), mortality risk increased three- to fourfold. Results from the SENECA study suggest, that even in older populations, dietary quality and lifestyle factors still have an effect on morbidity and mortality. Overall, a high-quality diet, nonsmoking, and physical activity in Europeans aged 70-75 showed a positive relationship to survival. Even more strongly related to survival was the combination of these healthy lifestyle factors. This information is extremely pertinent to prevention strategies aimed at older persons since these findings confirm that modifying lifestyle habits can positively influence mortality risk in older populations.

\section{The HALE study}

The Healthy Ageing: a Longitudinal study in Europe (HALE) comprised of individuals enrolled in the SENECA study and the Finland, Italy, the Netherlands, Elderly (FINE) study. ${ }^{16}$ This was a cohort study conducted from 1988 to 2000. Knoops and colleagues ${ }^{16}$ investigated the single and combined effect of dietary patterns and lifestyle factors (alcohol use, smoking status and physical activity level) with ten-year mortality from all causes, CHD, CVD, and cancer. The study population consisted of 1,507 seemingly healthy men and 832 women, aged 70-90 from 11 European countries. A low-risk group was defined for diet and lifestyle factors in order to assess the association with mortality. Consuming low quantities of alcohol per day, those who had never smoked or those who stopped smoking $>15$ years ago and for those who were at least minimally active and close to the Mediterranean dietary pattern, were considered as the low-risk group. Overall, 4 points could be obtained, 1 point for a Mediterranean diet, and 3 points for the other healthful lifestyle factors. Data analysis showed that northern Europe had a lower diet score than southern Europe; the mean alcohol consumption was $17.5 \mathrm{~g} /$ day and $5.5 \mathrm{~g} /$ day for men and women in northern Europe, respectively, compared to $31 \mathrm{~g} /$ day for men and $6 \mathrm{~g} /$ day for women, in southern Europe. During the 10-year follow-up, the number of men that died was double in comparison to the number of women. Analysis of the data associated physical activity, moderate alcohol use, nonsmoking and adherence to the Mediterranean type of diet, with a lower-risk of all-cause mortality. Those who had only one low-risk factor had a significantly greater risk of all-cause and cause-specific mortality compared to those who had two, three, or four factors. Not adhering to a low-risk pattern in 
cause-specific mortality was associated with $64 \%$ of deaths due to CHD and $61 \%$ due to CVD. Overall, those who had a combination of all four healthful diet and lifestyle factors had less than half the mortality rate from all causes, CHD, CVD, and cancer than those who did not. These findings support that a lower risk for all-cause and cause-specific mortality was associated with more healthful dietary and lifestyle factors in Europeans aged 70-90.

\section{The Framingham Heart Study}

During 1948 to 1951, the Framingham Heart Study (FHS) recruited 5,209 residents from Framingham, Massachusetts, aged 30-62. ${ }^{17}$ This sample, part of the original FHS, represented one-third of all Framingham residents in this age group at the time. Subjects were followed for more than 40 years and examined biennially for the development of CVD. The second cohort, the Framingham Offspring Study, included 3,544 children of parents that were in the original cohort, in addition to 1,580 spouses of these offspring. The final sample was 5,124 individuals that were enrolled from 1971 to 1978 . Allaire and colleagues ${ }^{18}$ used this data to examine evidence for secular change in disability and health among people aged 55 to 70 . Data analysis showed that CVD prevalence was $51 \%$ in the offspring cohort verses $79 \%$ in the original cohort. Secular trends from this study show that there is a decrease in some chronic diseases such as CVD, hypertension, and chronic obstructive pulmonary disease, although diseases such as obesity and diabetes are on the rise.

The diet in the FHS in comparison to the European studies was made by Haveman-Nies and colleagues ${ }^{19}$ who analyzed data from the Framingham and the European SENECA studies to evaluate the dietary quality of subjects in order to examine the relationship of these approaches to dietary and lifestyle factors. The combined study included 828 elders from the original Framingham cohort and the offspring cohort, where the age range of 70-77 years was matched to those of the SENECA participants. Data from the 1,282 Europeans enrolled in the SENECA study were also included. Two different assessment tools were employed to measure food and nutrient intake in the Framingham and SENECA studies. In order to compare food intake between the two studies, 12 food groups (grains, milk and milk products, fruit and fruit products, eggs, meat and poultry, fish/shellfish, vegetables, fat/oils, legumes/nuts/ seeds, sugar and sugar products, nonalcoholic and alcoholic beverages), based on the EUROCODE system, were defined for both studies. Data analyses revealed many disparities amongst subjects. In Framingham, energy intake was 7.4 MJ/day compared to $10.4 \mathrm{MJ} /$ day in Betanoz, Spain. Two main micronutrient profiles emerged in Europe: a Northern and Southern profile. The Northern profile had a similar contribution to total energy intake from fat and carbohydrates, whereas the Southern profile delivered a higher energy percentage from carbohydrates than fat. The Framingham elders were comparable to the Southern profile. In the southern European centers, elevated carbohydrate intakes were mainly attributable to high intakes of polysaccharides. Total fat intake in southern European centers consisted of minimum quantities of saturated fats and high amounts of monounsaturated fat compared to northern European centers. Results also found that elders from the Framingham study had higher vitamin intakes than the Europeans. Moreover, southern European centers were characterized by wine while in northern European centers, beer was more common. The following clusters were characteristic of particular locations; Cluster 1 was predominantly subjects from The Netherlands and Framingham and characterized by a high intake of sugar and sugar products, legumes/nuts/seeds, and a moderate intake of the other food groups. Participants from the southern European centers were part of cluster 2, which was characterized by high intakes of fish/shellfish and grains. The majority of persons from northern European centers were part of the third cluster characterized by high intakes of fat and of the food groups meat, poultry, and eggs. Women dominated the milk and fruit cluster and had high intake of vitamins and calcium, while men were mainly part of the alcohol cluster and had the lowest intake of vitamins and calcium. Mean diet scores were also more favorable in southern European centers compared to northern European centers. This study concluded that dietary scores and dietary clusters are complementary measures to classify dietary quality. Cluster analysis found that vast differences exist in food and nutrient intakes of Europeans and Americans.

\section{The European Prospective Investigation into Cancer and Nutrition Study (EPIC)}

The European Prospective Investigation into Cancer and Nutrition Study (EPIC) is a multinational, prospective cohort study that examines the role of biological, dietary, and environmental factors in cancer and other chronic diseases, coordinated by the International Agency for Research on Cancer. Participants from EPIC that were over the age of 60 , that had acceptable energy intakes, vital status, lifestyle and health variables were included in the EPIC-Elderly study database.$^{20}$ Consequently, 519,978 apparently healthy 
volunteers recruited between 1992 to 2000 from Denmark, France, Germany, Greece, Italy, the Netherlands, Norway, Spain, Sweden, and the United Kingdom were included in the EPIC-elderly study. This study investigated existing dietary patterns in European elders and how these patterns influenced their health and longevity. All participants were included in the EPIC-Elderly database, except those from Norway since the Norwegians included in the EPIC cohort were relatively young. Dietary intakes were collected by self- or interviewer-administered quantitative food frequency questionnaires. Lifestyle, anthropometric, and medical variables were also obtained. Data analysis showed that among European men aged 60 years or older, energy intake derived from carbohydrates was $45 \%$, from protein $15 \%$, from saturated lipids $13 \%$, from monounsaturated lipids $13 \%$, from polyunsaturated lipids $6 \%$, and from ethanol $5 \%$. Among women, total energy intake contribution from the same macromolecules was $46 \%, 16 \%, 13 \%, 13.6 \%$, and $3 \%$, respectively. Generally, vast differences existed in daily consumption of the designated food groups; however, within-center differences were small. Two main dietary profiles among Europeans emerged from the findings: a Northern and Southern profile. A Northern profile was characterized by a low consumption of fruits, pasta/rice/other grain, vegetables, bread, wine, legumes and vegetable oils, and a high consumption of butter, potatoes, dairy products and other cereals (flour, pastry, breakfast cereals), meat, non-alcoholic and alcoholic beverages (except wine), and sugar and cakes. On the contrary, high consumption of vegetables, fruits, vegetable oils, bread, pasta/rice/other grain, soups, legumes, fish and wine and low consumption of dairy products, nonalcoholic and alcoholic beverages, sugar and cakes were characteristic of the Southern profile. Nearly identical results were shown after multivariate analysis for male and female subjects; therefore, only findings referring to the total sample are stated. The first two patterns, of the seven identified, could be used to adequately describe the dietary habits of the EPIC-elderly participants. The first pattern reflects a vegetable-based diet characterized by a high consumption of vegetable oils, fruit, pasta, rice and other grain, vegetables and legumes (high positive scoring coefficients) and a low consumption of nonalcoholic beverages, potatoes, and margarine (negative scoring coefficients). The consumption of sweets and cereals, added fat and dairy (positive values) and the low consumption of bread, meat and eggs, and alcoholic beverages (negative values) reflect a sweet and fat-dominated diet. Multiple regression analysis for the vegetable-based pattern was positively associated with the female gender, higher educational level, total energy intake, physical activity, higher body mass index (BMI) and never and past smoking. Age and waist-to-hip ratio were inversely associated with this pattern. Moreover, the first pattern was more frequent in participants living in southern Europe and decreased in northern countries with the exception of the Oxford health-conscious participants. Additionally, three major clusters were derived; Cluster A (primarily participants from Italy, Spain, and Greece) was characterized by a high consumption of vegetables, legumes, fruit, pasta, rice and other grain, vegetable oils, wine and a low consumption of potatoes, butter, margarine, dairy products, meat, sugar, cakes, nonalcoholic and alcoholic beverages except for wine. A high consumption of potatoes, other cereals, butter, margarine, sugar and cakes, and a low consumption of vegetables, legumes, pasta, rice and other grains, fruit and vegetables oils characterized cluster B. Cluster C was very similar to cluster B; however, consumption of other cereals, butter, margarine, sugar and cakes was lower in cluster $\mathrm{C}$. The consumption of eggs, nonalcoholic and alcoholic beverages (except for wine) was higher in cluster C than in cluster B. Sweden and Denmark's elderly population belonged primarily to cluster $\mathrm{B}$ and $\mathrm{C}$, respectively. Participants from France and northern Europe were grouped into clusters B and C in moderately similar proportions. From this study dietary patterns of Europeans, aged 60 and older, from nine different European countries emerged. These findings are essential in understanding existing dietary patterns in Europe and identifying morbidity and mortality risk.

\section{The INTERHEART study}

The INTERHEART study is a multicenter case-control study that investigates the relationship between protective or risk factors to acute myocardial infarction. ${ }^{3}$ Study participants (15,152 cases and 14,820 controls) were recruited from 52 countries in Asia, Europe, the Middle East, Africa, Australia, North America, and South America. The overall median age was 58 years. At minimum, one age-matched ( \pm 5 years) and sex-matched control was recruited per case. The same exclusion criteria applied to controls and cases, with the exemption that controls had no previous diagnosis of heart disease or history of chest pain. Information on demographic factors, socioeconomic status, lifestyle habits, personal and family history of CVD, psychosocial factors, anthropometrical measurements and non-fasting blood samples were obtained. Details on inclusion criteria, study procedures and statistical analysis were recorded elsewhere. ${ }^{3}$ Data analyses suggested that all risk factors (smoking, diabetes, 
hypertension, abdominal obesity, psychosocial, vegetables and fruit daily, exercise, alcohol, apolipoproteins B and A1) were significantly ( $p<0.0001$ ) related to acute myocardial infarction, apart from alcohol which had a weaker association $(p=0.03)$. The two strongest risk factors were current smoking and raised apolipoproteins B and A1, followed by history of diabetes, hypertension and psychosocial factors. Protective factors included daily consumption of fruits or vegetables, moderate or strenuous physical exercise, and consumption of alcohol three or more times a week. Daily consumption of fruit and vegetables and regular physical activity gave an odds ratio of 0.60 (99\% CI: $0.51-0.71)$ and if an individual avoided smoking the odds ratio would decrease to 0.21 . This suggests that a healthy lifestyle can possibly reduce the risk of acute myocardial infarction by more than three-quarters in comparison with a smoker with poor lifestyle. Abnormal lipids had the highest populationattributable risk (PAR) in men (49.5\%) and women (47.1\%), which was the most important risk factor with respect to PAR in both young and old persons. This study revealed that these nine risk factors accounted for a substantial proportion of the risk for a first acute myocardial infarction. Furthermore, across all geographic regions the two most important risk factors were smoking and abnormal lipids. Combined, these factors accounted for nearly two-thirds of the PAR of an acute myocardial infraction. These findings suggest that smoking cessation and modification of lipid abnormalities can greatly reduce the risk of myocardial infarction. Consistent fruit and vegetable consumption was associated with a $30 \%$ relative risk reduction and when combined with exercise and smoking avoidance it could lead to an approximate $80 \%$ lower relative risk of myocardial infarction. This substantiates the evidence that lifestyle modification, especially smoking avoidance, high fruit and vegetable consumption, and adequate exercise, should be the foundation of worldwide prevention efforts that aim to decrease risk of CVD. Overall, this study showed that nine potentially modifiable risk factors were associated to more than $90 \%$ of the risk of an acute myocardial infarction. Moreover, since this study included a vast number of individuals and varying ethnic groups from all geographic regions of the globe, results suggest that preventative methods to myocardial infarction could be applicable worldwide.

\section{The Mediterranean Islands Study (MEDIS)}

The Mediterranean Islands Study (MEDIS) examined bioclinical, lifestyle, behavioral, and dietary characteristics in a sample of elderly people living in the Mediterranean Islands. ${ }^{21}$
MEDIS is a health and nutrition survey where a random, population-based, multistage sampling method was used to select 1,190 men and women ( $>65$ years) from the Republic of Cyprus and the following Greek islands: Mitilini, Samothraki, Cephalonia, Crete, Limnos, Corfu, and Zakynthos. Individuals who resided in assisted-living centers and those with history of CVD or cancer were excluded. The MedDietScore ${ }^{21}$ (range 0-55) was used to assess the level of adherence to the Mediterranean diet. Fasting plasma glucose tests determined diabetes mellitus status, which was analyzed in accordance with the American Diabetes Association diagnostic criteria. Fasting blood lipids levels, high-density lipoprotein-cholesterol (HDL-C), low-density lipoprotein-cholesterol (LDL-C) and triglycerides were recorded. Hypercholesterolemia was defined by the use of lipid-lowering agents or total serum cholesterol levels $>200 \mathrm{mg} / \mathrm{dl}$.

An analysis of the sub-group of elderly Cypriots of the MEDIS study showed that, after controlling for age, sex, body mass index, smoking and physical activity status, greater adherence to the Mediterranean diet was associated with a 23\% lower likelihood of having hypercholesterolemia. ${ }^{22}$ A $26 \%$ reduction was associated with the combination of diet and statin treatment. Moreover, MEDIS investigators revealed an association between long-term fish intake and health status. ${ }^{23}$ Particularly, after adjusting for various variables, fish intake was inversely associated with systolic blood pressure $(\mathrm{p}=0.026)$, fasting glucose $(\mathrm{p}<0.001)$, total serum cholesterol $(\mathrm{p}=0.012)$, and triglyceride levels $(\mathrm{p}=0.024)$. Additionally, multinomial logistic regression revealed that a reduction of $100 \mathrm{~g}$ per week in fish intake was associated with a 19\% higher likelihood of having one additional cardiovascular risk factor such as hypertension, hypercholesterolemia, diabetes or obesity. MEDIS investigators also evaluated whether alcohol consumption is associated to blood pressure in the sample of elderly men and women. ${ }^{24}$ Data analysis revealed that $44 \%$ of men and $19 \%$ of women consumed alcohol no less than once a week; where red or white wine and retsina, a locally produced alcohol beverage, were primarily consumed. Subjects reported this drinking pattern for at least the past 10 years. A semiparabolic relationship was observed between alcohol consumption and all blood pressure measurements. Specifically, when adjusting for confounding factors (age, sex, years of school, physical activity level, body mass index, medication use, dietary and smoking habits) a J-shaped association of alcohol intake with systolic $(\mathrm{p}=0.001)$, diastolic $(\mathrm{p}=0.02)$, mean $(\mathrm{p}=0.001)$, and pulse pressure $(\mathrm{p}=0.07)$ was found. Therefore, long-term moderate alcohol consumption in elders can improve blood pressure levels and thus, CVD prognosis. 
Lastly, the association between the consumption of various food patterns and the prevalence of CVD risk factors among elders was also evaluated in the MEDIS study. ${ }^{25}$ Principal components analysis was performed to obtain food patterns. Predominantly, participants were characterized by virtually daily use of olive oil, fruits, vegetables, salads and greens. Meat (red or white) was consumed frequently and there was a moderate intake of alcohol. Five components were extracted, which explained $56.53 \%$ of the total variation in intake. The following food patterns were developed: component 1 was heavily loaded by the intake of low-fat products (fish, vegetables, legumes, greens, salads) and olive oil; component 2 included a high glycemic index and high-fat pattern (red or white meat and meat products, pasta and potatoes); component 3 included consumption of cereals and sweets; component 4 was characterized by dairy products and fruit consumption; component 5 was characterized by the intake of alcoholic beverages. Component 1 (mainly low fat products and olive oil) was inversely associated with age $(p=0.001)$, positively associated with the male sex $(\mathrm{p}=0.05)$ and physical activity status $(\mathrm{p}=0.001)$. Furthermore, it was associated with $9.2 \%$ less likelihood of having diabetes. Component 2 (mainly high fat foods) was positively associated with the male sex $(p=0.04)$ and with $4.8 \%$ higher likelihood of having hypercholesterolemia $(p=0.06)$. Component 3 (characterized by cereal intake) was positively associated with age $(\mathrm{p}=0.008)$, income $(\mathrm{p}=0.001)$, and physical activity level $(\mathrm{p}=0.009)$. This pattern was also associated with $28.4 \%$ lower likelihood of having hypercholesterolemia $(\mathrm{p}=0.001)$, $25 \%$ lower likelihood of having diabetes $(\mathrm{p}=0.009)$ and $33 \%$ lower likelihood of being obese $(\mathrm{p}=0.001)$. Component 4 was positively associated with age $(p=0.042)$ and smoking habits $(\mathrm{p}=0.009)$. Lastly, component 5 , was positively associated with the male sex $(p=0.001)$, age $(p=0.003)$, income $(p=0.013)$, and smoking $(p=0.049)$. The other two components were not associated with the CVD risk factors investigated in the MEDIS study.

\section{The Health Professional Follow-Up Study}

The Health Professional Follow-up Study (HPS) commenced in 1986, and the association between dietary patterns and risk for type 2 diabetes was examined in 42,504 predominately white men (40-70 years of age). ${ }^{26}$ Men were excluded if they reported having diabetes, CVD or cancer at baseline. Information on diet was collected using a 131-item semiquantitative food frequency questionnaire. Every two years participants were mailed a questionnaire for updated information. Factor analysis was applied to assess dietary patterns. Lifestyle information, family history of diabetes, and medical history were also recorded. Men that fit the 1985 WHO criteria for diabetes were excluded. A supplementary questionnaire was received biennially regarding symptoms, diagnostic tests and medication use in order to confirm diagnosis of diabetes mellitus. Analysis revealed one factor (labeled the prudent dietary pattern) which was characterized by high consumption of vegetables, legumes, fruit, whole grains, fish and poultry. Red meat, processed meat, refined grains, french fries, high-fat dairy products, sweets and desserts, high-sugar drinks and eggs characterized the western dietary pattern. A higher prudent-pattern score was associated with high intakes of protein, cereal fiber and magnesium in addition to low intakes of alcohol, saturated fat and total fat. Moreover, men that fit that dietary pattern were older, more physically active, less likely to be smokers and more likely to have hypercholesterolemia (hypercholesterolemia may reflect changes in diet after diagnosis). On the contrary, a western pattern score was associated with higher intakes of fat and alcohol and lower intakes of cereal fiber and magnesium. Additionally, these men had a higher BMI, low physical activity, less likely to have hypercholesterolemia and more likely to be smokers and of northern European ancestry. The prudent pattern was associated with a modest risk reduction for type 2 diabetes (multivariate relative risk for highest vs lowest quintiles, 0.84; 95\% CI: 0.70-1.00) whereas the higher western-pattern scores were associated with a considerable increased risk for type 2 diabetes (relative risk, 1.59; 95\% CI: 1.32-1.93; $\mathrm{p}<0.001)$. Moreover, when examining specific foods that characterized the prudent diet, a strong inverse association existed with whole grains and risk for type 2 diabetes. Unprocessed meat, processed meat, refined grains and high-fat dairy products were specific foods with major contributions to the western pattern. They were all positively associated with risk of type 2 diabetes. For subgroups separated by age, family history of diabetes, BMI, and physical activity a consistent association with type 2 diabetes existed. Lastly, obese men in the highest quintile of the western dietary-pattern score had the highest risk for type 2 diabetes. Study findings confirm that dietary patterns can predict risk for type 2 diabetes. The western dietary pattern was substantially associated with type 2 diabetes and may influence CVD incidence as diabetes is a common risk marker.

\section{A summary of clinical trials Indo-Mediterranean Heart study}

People of South Asian origin have greater susceptibility to CVD that cannot be explained by conventional risk factors 
such as obesity and cholesterol. As a result, a randomized, single-blind trial to assess the cardio-protective effects of a Mediterranean-type diet in middle-aged and elderly patients with angina, pectoris, myocardial infarction, or other risk factors for coronary artery disease (CAD) was completed. ${ }^{27}$ Patients were randomized into two groups: 499 cases which adopted a diet rich in whole grains, fruits, vegetables, walnuts, and almonds and 501 controls which consumed a local diet similar to the Step I National Cholesterol Education Program (NCEP) prudent diet. Baseline dietary intake was determined by meeting weekly for three weeks. Daily diet, including weights of fruit, vegetables, and nuts, as well as drug intake was recorded. Additionally, during these three weeks, baseline clinical, electrocardiographic, radiological, and laboratory data were obtained from the participants. Both groups consisted of two-thirds vegetarian patients. Moreover, they were advised to substitute foods that provide similar dietary intake to the step 1 NCEP prudent diet - less than $30 \%$ of energy from total fat, $<10 \%$ from saturated fat, $<300 \mathrm{mg}$ cholesterol per day. The intervention group was also recommended to eat at least $400-500 \mathrm{~g}$ of fruits, vegetables, and nuts per day, in addition to $400-500 \mathrm{~g}$ of whole grains daily and 3-4 servings of mustard seed or soy bean oil per day. These dietary recommendations aimed to provide ample phytochemicals, antioxidants and $\alpha$-linolenic acid, which are in accordance with WHO and the Indian Consensus Group to prevent chronic disease. Cardiovascular endpoints were nonfatal myocardial infarctions, sudden cardiac deaths, and the combined total of these events. Data analyses showed that after the two-year follow-up, an increase in fruit, vegetables, legumes, and oils was observed in both groups. Lifestyle habits did not differ significantly between groups. Baseline results did not show considerable differences in carbohydrate, fatty acid and cholesterol consumption between groups. After the two-year follow-up, omega-3 fatty acid intake was higher in the controls than the intervention arm. Both groups had similar fiber, salt, fruit, vegetable, nut, and whole grain intakes. However, after two years, the intervention group consumed a greater percentage of calories from total and complex carbohydrates, had higher polyunsaturated to saturated fat ratio, and lower energy, total fat, saturated fat, and cholesterol than did the controls. Total fruit, vegetable, nut, whole grain, and $\alpha$-linolenic acid intakes as well as mustard and soy oil consumption were significantly higher in patients on the intervention diet. Both groups had a significant decrease in total and LDL-C and triglycerides. The reduction was greater in the intervention group than in the controls. HDL increased in the intervention arm, but decreased in the controls. A significant reduction was observed in fasting blood glucose, BMI, and blood pressures in the intervention arm. Smoking in both groups had a significant reduction. A significant reduction in risk of nonfatal myocardial infarction, sudden cardiac death, and total cardiac endpoints was observed in the intervention group. There were also less fatal myocardial infarctions, but not significantly fewer. Cardiac complications such as angina pectoris, left ventricular strain, and arrhythmias requiring treatment were also significantly less common in the intervention group. The effect of the intervention was not determined by pre-existing conditions at baseline. Interactions were tested with respect to $\mathrm{CAD}$, artery disease, hypertension and hypercholesterolemia and found none were significant. Results from this study suggest that an Indo-Mediterranean diet, rich in $\alpha$-linolenic acid, is associated with a significant reduction in CAD morbidity and mortality. Moreover, results showed greater improvements in surrogate traditional risk factors in those adhering to the intervention diet than the prudent Step I diet.

\section{The Mediterranean Diet, Cardiovascular Risks and Gene Polymorphism Study}

This trial examined the effects of a Mediterranean-type diet compared to a low-fat diet on CVD risk. Subjects were middle-aged and elderly that were visiting the Center for Detection and Prevention of Arteriosclerosis (CDPA) and were at moderate CVD risk. ${ }^{28}$ At baseline, participants were eating a diet similar to a western-type diet. As part of the three-month nutritional strategy, subjects were provided with information on the recommended Mediterranean diet (adapted from the traditional model) or the low fat-diet (the low-fat American Heart Association-type diet adapted by the CDPA). The two diets were similar in terms of overall nutrients; except for the quantity and quality of lipid intake, which was greater in the Mediterranean-type diet. Compliance to the recommended diets was verified by three-day food records and 24-hour unscheduled dietary recalls. Analysis included 102 subjects in the Mediterranean diet group and 110 in the low-fat group. The mean age was $50.8( \pm 10.8)$ and $51.6( \pm 10.3)$ in each group, respectively. After three months, energy intake and ingested cholesterol decreased in both groups. Energy provided by proteins and carbohydrates significantly increased in both groups while that provided by total fat decreased. The percentage of energy provided by saturated fat decreased throughout the intervention period whereas polyunsaturated fat increased. Plasma total cholesterol significantly decreased after the 
three-month intervention, with a tendency for a group effect $(\mathrm{p}=0.082)$ in the Mediterranean diet group $(-0.4 \mathrm{mmol} / \mathrm{L}$, or $-7.5 \%)$ and in the low-fat group $(-0.3 \mathrm{mmol} / \mathrm{L}$, or $-4.5 \%)$. No change was observed in HDL-C however, triglyceriderich lipid cholesterol decreased significantly in both groups. In LDL-C there was a trend $(\mathrm{p}=0.09)$ for a time-by-group interaction and a trend for a main effect of group $(\mathrm{P}=0.074)$ : Mediterranean diet group ( $-0.05 \mathrm{mmol} / \mathrm{L})$ and low-fat group $(-0.2 \mathrm{mmol} / \mathrm{L})$. In both groups, plasma triacylglycerol and triglyceride-rich lipid triacylglycerol concentrations significantly dropped after three months. Similarly, apolipoprotein-A1 and apolipoprotein-B were significantly lower. Overall, findings displayed a greater reduction in LDL-C in the Mediterranean diet compared to a low-fat diet. Nevertheless, both diets displayed a measurable efficiency in reducing risk factors for CVD.

\section{The Lyon Diet Heart Study}

The Lyon clinical trial tested the protective effect of a Mediterranean-type diet on reducing the risk of cardiovascular complications after a first myocardial infarction. ${ }^{29,30}$ This was a randomized single-blind trial with a two-step recruitment procedure. Patients who agreed to be followed up for five years were assigned to a control or study group. The study included 605 middle-aged and elderly patients that were randomized into two groups. The first group adopted a Mediterranean diet, whereas the control group did not receive any further information. The Mediterraneantype diet tested supplied $<35 \%$ of energy from fat, $<10 \%$ of energy from saturated fat, $<4 \%$ of energy from linoleic acid and $>0.6 \%$ of energy from $\alpha$-linolenic acid. Both groups attended annual visits where they completed a clinical examination by a cardiologist, electrocardiogram and blood sampling for routine biological measurements. These annual visits did not replace regular visits to attending physicians. The study and control groups were used to observe and compare correlations to primary and secondary end-points. Cardiovascular death and nonfatal acute myocardial infarction were primary endpoints. Major secondary endpoints were unstable angina and stroke whereas minor secondary endpoints consisted of myocardial revascularization (bypass surgery). The protective role of cardiac drugs (anticoagulants agents, aspirin, blocking agents and calcium channel-blocking agents and inhibitors) was also tested. Regarding medication, a parallel course was followed in the two groups. Aspirin was the sole factor significantly associated with new events. Analysis showed that for primary and secondary end-points combined, 59 events occurred in the control and 14 in the study group (risk ratio, 0.24; 95\% CI: $0.13-0.44$; $p<0.0001)$. This was a 76\% reduction in risk. For primary, major secondary and minor secondary end-points combined, 104 events occurred in the control group and 68 events in the study group (risk ratio, $0.63 ; 95 \%$ CI: $0.46,-0.87 ; p<0.005)$. Due to the statistically significant results from this study, a decision was made to stop the trial. Results suggest that that underlying pathogenic mechanisms of varying cardiac events are influenced differently by the nutrient properties of the diet. For example, large differences existed between the control and study groups in episodes of new infractions and unstable angina. This is not surprising as they both have similar underlying pathogenic mechanisms. Furthermore, this study supports that dietary modification, such as adopting a Mediterranean-type diet, can interfere with the occurrence of acute coronary events. Additionally, the final report of the Lyon trial presented a follow-up (mean of 46 months per patient) which dealt with the relationships of dietary patterns and traditional risk factors with reoccurrence. ${ }^{30}$ Results examining the relationship of the Mediterranean dietary pattern and re-occurrence found that the rate of cardiac death and nonfatal infarction in the experimental group after 46 months (1.24/hundred patients/year) was similar to the observations made after 27 months (1.32). The protective effect of the Mediterranean diet was confirmed by findings that determined the rate in control subjects to be 4.07 after 46 months, versus 5.55 after 27 months. Findings from this trial substantiate the cardioprotective effect of dietary modification and confirm that long-term compliance to new dietary habits is possible.

\section{Discussion and conclusions}

Lifestyles and demographics are changing worldwide prompting shifts in food consumption patterns and disease trends. However, while dietary patterns have been linked to CVD risk, ${ }^{3,13-16,31}$ the most effective and efficient method of overcoming the multiple factors that hinder healthy eating amongst elders has yet to be determined. Yusuf and colleagues ${ }^{32}$ describe the various obstacles faced by developing countries that contribute to CVD burden: uncontrolled growths of cities in developing countries lead to longer commutes for workers with trains and buses and infringement of public spaces, such as parks and walking paths, in the interest of commercialization has led to a decrease physical activity. Also, availability, ${ }^{33}$ familiarity to certain foods, ${ }^{34-36}$ advertising, ${ }^{37}$ and westernization ${ }^{38}$ are a few obstacles that elders face. There is a need to understand how evidence-based science can parallel the realities facing most elders. 
The summarized findings revealed that many food choices might reduce CVD risk in the elderly. For example, a dietary pattern like the Mediterranean diet seems protective in elder people's cardiac health. High intakes of vegetables, fresh fruits, cereals, and olive oils and low intakes of saturated fats are defining characteristics of the Mediterranean dietary pattern. It has been suggested that this dietary pattern may prevent lipid abnormalities, systolic and diastolic blood pressure rise, arrhythmias, diabetes and obesity incidence, and some types of psychological disorders (depression) through the amelioration of insulin sensitivity and their anti-inflammatory and antioxidant actions. ${ }^{39,40}$ Moreover, increased abdominal fat accumulation has been linked to the progression of insulin resistance. Adipose tissue is recognized as a highly active metabolic and endocrine organ that secretes fatty acids. These nonesterfied fatty acids inhibit insulin-stimulated glucose metabolism and stimulate gluconeogenesis in the liver. Consequently, dietary patterns, such as the Mediterranean diet that promote weight maintenance may protect against the development of diabetes. In addition results from the ATTICA study, which included 3,042 middle-aged men and women from Greece, showed that greater adherence to the Mediterranean diet was associated with increased total antioxidant capacity and decreased oxidized LDL levels. ${ }^{41}$ Oxidized LDL levels are believed to play a biological role in the early development of atherosclerotic lesions. Thus, as a result, the Mediterranean diet may also, partially, reduce CVD risk due to its ability to protect individuals from oxidative stress.

Efforts are needed to produce a cardioprotective diet that generates the maximum reduction in CVD risk in older populations. Additionally, due to the limited available studies that investigate diet and CVD risk in elders, findings from middle-aged populations steer older adult guidelines. Studies need to investigate effects of diets, especially, in all elderly populations older than 65 years and the oldest elderly ( $85+$ years).

As a result, nutritionists and public health policy makers need to address the barriers that hinder healthy eating in older populations and identify the factors facilitating unhealthy food consumption. Health messages should not only focus on which foods to avoid, but need to provide unambiguous guidance on how to prepare cost-effective meals that meet dietary recommendations. ${ }^{42}$ More research is needed to understand the effect of healthy diets and lifestyles on global health in older populations. In addition, much effort is needed to determine effective ways to modify food habits, especially in developing countries. Intervention strategies aimed at preventing morbidity and premature mortality in older people, worldwide, need to reflect the evidence-based science from studies examining dietary habits, including various physical, psychological, and environmental factors that influence consumer food choices.

\section{Disclosure}

The authors report no conflicts of interest in this work.

\section{References}

1. World Health Organization. Report of the World Health Organization Study Group. Diet, Nutrition and the Prevention of Chronic Diseases. 2008. Accessed December 15, 2009. Available from: http://whqlibdoc. who.int/trs/WHO_TRS_916.pdf.

2. World Health Organization. Keep Fit For Life. Meeting the nutritional needs of older persons. Geneva, Switzerland: WHO; 2002.

3. Yusuf S, Hawken S, Ounpuu S, et al. Effect of potentially modifiable risk factors associated with myocardial infarction in 52 countries (the INTERHEART study): case-control study. Lancet. 2004;364:937-952.

4. World Health Organization. Prevention of Cardiovascular Disease: Pocket guidelines for assessment and management of cardiovascular risk. Geneva, Switzerland: WHO; 2007.

5. European Cardiovascular Disease Statistics. British Heart Foundation Health Promotion Research Group and Health Economics Research Centre. 2005 edition.

6. World Health Organization. Active Ageing: A Policy Framework 2002. Accessed January 14, 2009. Available from: http://whqlibdoc. who.int/hq/2002/WHO_NMH_NPH_02.8.pdf.

7. Fung J, Maggi S, Steel K. For the good health of senior citizens. World Health Forum. 1993;14:145-156.

8. Kinsella K, Velkoff VA. An Aging World: 2001. Washington, DC: US Government Printing Office; 2001.

9. Kris-Etherton P, Eckel R, Howard B, Jeor S, Bazzare T. Lyon diet heart study: Benefits of Mediterranean-Style, National Education Program/ American Heart Association Step 1 Dietary Pattern on Cardiovascular Disease. Circulation. 2001;103(13):1823-1825.

10. Lichtensein A, Appel L, Brands M, et al. Summary of American Heart Association Diet and Lifestyle Recommendations Revision 2006. Arterioscler Thromb Vasc Biol. 2006;26:2186-2191.

11. Tufts University Homepage. Massachusetts: Tufts University. 2007. Accessed November 12, 2008. Available from: http://nutrition.tufts. edu/docs/pdf/releases/ModifiedMyPyramid.pdf.

12. Panagiotakos D. $\alpha$-priori versus $\alpha$-posterior methods in dietary pattern analysis: a review in nutrition epidemiology. British Nutrition Foundation Nutrition Bulletin. 2008;33:311-315.

13. Willet WC. The Mediterranean diet: science and practice. Public Health Nutr. 2006;9:105-110.

14. Menotti A, Kromhout D, Blackburn H, et al. Food intake patterns and 25-year mortality from coronary heart disease: Cross-cultural correlations in the Seven Countries Study. Eur J Epidemiol. 1999;15:507-515.

15. Haveman-Nies A, de Groot LC, Burema J, et al. Dietary Quality and lifestyle factors in relation to 10-year mortality in older Europeans - The SENECA Study. Am J Epidemiol. 2002;156:962-968.

16. Knoops K, de Groot LC, Kromhout D, et al. Mediterranean Diet, Lifestyle Factors, and 10-year Mortality in Elderly European Men and Women. J Am Med Assoc. 2004;292(12):1433-1439.

17. Hubert H, Feinleib M, McNamara PM, Castelli WP. Obesity as an independent risk factor for cardiovascular disease: A 26-year follow-up of participants in the Framingham Heart Study. Circulation. 1983;67(5):968-977.

18. Allaire SH, LaValley MP, Evans SR, et al. Evidence for decline in disability and improved health among persons aged 55 to 70 years: The Framingham Heart Study. Am J Public Health. 1999;89:1678-1683. 
19. Haveman-Nies A, Tucker KL, de Groot LC, Wilson PWF, van Staveren WA. Evaluation of dietary quality in relationship to nutritional and lifestyle factors in elderly people of the US Framingham Heart Study and the European SENECA study. Eur J Clin Nutr. 2001;55:870-880.

20. Bamia C, Orfanos P, Ferrari P, et al. Dietary patterns among older Europeans: the EPIC-Elderly study. Br J Nutr. 2005;94:100-113.

21. Tyrovolas S, Polychronopoulos E, Bountziouka V, et al. Level of adherence to the mediterranean diet among elderly individuals living in Mediterranean islands: Nutritional Report from the MEDIS Study. Ecol Food Nutr. 2008;48:76-87.

22. Polychronopoulos E, Panagiotakos D, Polystipioti A. Diet, lifestyle factors and hypercholesterolemia in elderly men and women from Cyprus. Lipids Health Dis. 2005;4:17.

23. Panagiotakos D, Zeimbekis A, Boutziouka V, et al. Long-term fish intake is associated with better lipid profile, and arterial blood pressure, blood glucose levels in elderly people from Mediterranean islands (MEDIS epidemiological study). Med Sci Monit. 2007;13(7):307-312.

24. Panagiotakos D, Kourlaba G, Zeimbekis A, Toutouzas P, Polychronopoulos E. The J-shape association of alcohol consumption on blood pressure levels, in elderly people from the Mediterranean Islands (MEDIS epidemiological study). J Hum Hypertens. 2007;21(7): 585-587.

25. Panagiotakos D, Bountziouka V, Zeimbekis A, Vlachou I, Polychronopoulos E. Food pattern analysis and prevalence of cardiovascular disease risk factors among elderly people from Mediterranean islands. J Med Food. 2007;10(4):615-622.

26. van Dam RM, Rimm EB, Willet WC, Stampfer M, Hu F. Dietary patterns and risk for type 2 diabetes mellitus in U.S. men. Ann Intern Med. 2002;136:201-209.

27. Singh RB, Niaz MA, Ghosh S, et al. Effect of an Indo-Mediterranean diet on progression of coronary artery disease in high risk patients (Indo-Mediterranean Diet Heart Study): a randomized single-blind trial. Lancet. 2002;360:1455-1461.

28. Vincent-Baudry S, Defoort C, Gerber M, et al. The Medi-RIVAGE study: reduction of cardiovascular disease risk factors after 3-mo intervention with a Mediterranean-type diet or low-fat diet. Am J Clin Nutr. 2005;82:964-971
29. De Lorgeril M, Salen P, Martin JL, et al. Effect of a Mediterranean type of diet on the rate of cardiovascular complications in patients with coronary artery disease. J Am Coll Cardiol. 1996;28:1103-1108.

30. De Lorgeril, Salen P, Martin JL, Monjaud I, Delaye J, Mamelle N. Mediterranean diet, traditional risk factors, and the rate of cardiovascular complications after myocardial infarction: Final Report of the Lyon Diet Heart Study. Circulation. 1999;99:779-785.

31. Sofi F, Cesari F, Abbate R, Gensini GF, Casini A. Adherence to Mediterranean diet and health status: meta-analysis. $\mathrm{Br} \mathrm{Med} J$. 2008;337:1344-1351.

32. Yusuf S, Reddy S, Ỏunpuu S, Anand S. Global burden of cardiovascular diseases. Circulation. 2001;104:2746-2753.

33. McKie L. Older people and food: independence, locality and diet. Br Food J. 1999;101(7):528-536

34. Sulander T, Helakorpi S, Rahkonen O, Nissinen A, Uutela A. Changes and associations in healthy diet among the Finnish elderly, 1985-2001. Age Ageing. 2003;32:394-400.

35. Gustafsson K, Sidenvall B. Food - related health perceptions and food habits among older women. J Adv Nurs. 2002;39(2):164-173.

36. Johansson L, Thelle DS, Solvoll K, Bjørneboe GE, Drevon CA. Healthy dietary habits in relation to social determinants and lifestyle factors. Br J Nutr. 1999;81:211-220.

37. Lupton D. Food, the Body and the Self. London: Sage Publications; 1996.

38. Woo KS, Chook P, Raitakari OT, McQuillan B, Feng JZ, Celermajer DS. Westernization of Chinese adults and increased subclinical atherosclerosis. Arterioscler Thromb Vasc Biol. 1999;19:2487-2493.

39. Panagiotakos DB, Tzima N, Pitsavos C, et al. The relationship between dietary habits, blood glucose and insulin levels among people without cardiovascular disease and type 2 diabetes; the ATTICA study. Rev Diabet Stud. 2005;2:208-215.

40. Schröder H. Protective mechanism of the Mediterranean diet in obesity and type 2 diabetes. J Nutr Biochem. 2007;18:149-160.

41. Pitsavos C, Panagiotakos DB, Tzima N, et al. Adherence to the Mediterranean diet is associated with total antioxidant capacity in healthy adults: the ATTICA study. Am J Clin Nutr. 2005;82:694-699.

42. Popkin B, Haines P, Patterson R. Dietary changes in older Americans, 1977-1987. Am J Clin Nutr. 1992;55:823-830.
Clinical Interventions in Aging

\section{Publish your work in this journal}

Clinical Interventions in Aging is an international, peer-reviewed journal focusing on evidence-based reports on the value or lack thereof of treatments intended to prevent or delay the onset of maladaptive correlates of aging in human beings. This journal is indexed on PubMed Central, MedLine, the American Chemical Society's 'Chemical

\section{Dovepress}

Abstracts Service' (CAS), Scopus and the Elsevier Bibliographic databases. The manuscript management system is completely online and includes a very quick and fair peer-review system, which is all easy to use. Visit http://www.dovepress.com/testimonials.php to read real quotes from published authors. 